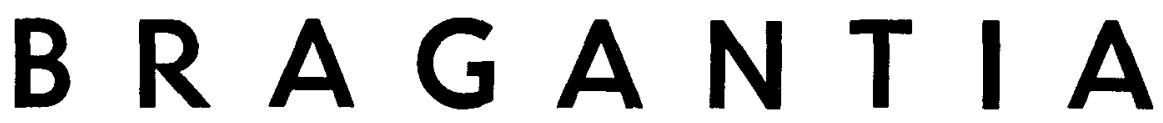

Revista Científica do Instituto Agronòmico do Estado de Sāo Paulo

Vol. 35

Campinas, agosto de 1976

N. ${ }^{\circ} 23$

\title{
EFEITOS DO BORO EM CANA-DE-AÇUCAR CULTIVADA EM VASOS CONTENDO SOLO ${ }^{(1)}$
}

A. Espironelo $\left({ }^{2}\right)$, Seção de Cana-de-Açúcar, Instituto Agronômico, M. O. C. Do BRAsIl Sobrinho, Departamento de Solos e Geologia e R. S. DE MORAES, Departamento de Matemática, E. S. A. "Luiz de Queiroz", USP

\section{SINOPSE}

Em ensaios em vasos foram estudados os efeitos do boro na cana-de-açúcar var. CB 41-14 (cana-planta e cana-soca), através da administração de doses crescentes do elemento à cana-planta cultivada em solo da série Ibitiruna, do Município de Piracicaba, com teor muito baixo de boro solúvel em água quente. Ao solo dos vasos (80 litros de capacidade) foram administradas as doses de $0,0,5$, $1,2,3,4$ e $8 \mathrm{ppm} \mathrm{B}$, sob a forma de ácido bórico.

Foram obtidos dados de produção de cana (planta e soca) e do teor de boro em folhas de três posições na planta $(+3,+1$ e -1$)$, em amostragens feitas aos cinco e oito meses de idade da cana-planta, e calcularam-se dados de açúcar-provável (cana-planta e soca).

O boro aplicado ao solo não proporcionou aumentos nas produções de cana e de açúcar-provável, tanto em cana-planta como em cana-soca, induzindo, entretanto, efeito depressivo em ambos os casos, pela dose mais elevada (8 ppm B) na cana-planta. Os teores de $B$ nas folhas $(+3,+1$ e -1$)$ de cana-planta, aos cinco meses de idade, cresceram com as doses administradas ao solo, alcançando níveis muito elevados $(289 \mathrm{ppm})$ no tratamento $8 \mathrm{ppm} \mathrm{B}$, com a média geral atingindo $124,4 \mathrm{ppm}$ B. Aos oito meses, os valores foram bem mais baixos (média geral $61,4 \mathrm{ppm}$ B) e nāo seletivos em relação à dose do boro aplicada. A melhor época de amostragem estaria em torno de cinco meses. As três posições de folha estudadas mostraram-se eficientes em relação às doses de boro aplicadas ao solo, sendo que +3 e +1 deram valores mais altos do que -1 .

(1) Pesquisa realizada com ajuda financeira da FAPESP. Recebida para publicação em 6 de fevereiro de 1976.

(2) Com bolsa de suplementação do C.N.Pq. 


\section{1 - INTRODUÇÃO}

Tanada \& Dean (17), mediante análise de tecidos, estudaram comparativamente a distribuição de $\mathbf{B}$ na cana-de-açúcar com quinze meses de idade e que vegetou em dois tipos de solos $(0,8$ e $1,8 \mathrm{ppm}$ de $\mathrm{B}$ solúvel em água) e em solução nutritiva completa e sem B. Encontraram, para os solos, de 1,5 a 9,5 ppm de B em diferentes folhas; de 2,4 a 11,0 para a solução completa e de 1,3 a 3,1 , para a solução completa menos boro. Calcularam tais autores que a cana, em cultura normal, retira do solo $0,5 \mathrm{~kg} / \mathrm{ha}$ de $\mathrm{B}$.

Ayres (1) não observou efeito benéfico à cana quando aplicou silicato de cálcio que continha $0,04 \%$ de $\mathrm{B}$ em vasos contendo solo.

Bowen (2) observou no meristema da cana-de-açúcar, variação de 22 a $57 \mathrm{ppm}$ de B numa variedade, e de 11 a $36 \mathrm{ppm}$ em outra, dos 3 aos 8 meses. Na segunda fase de sua pesquisa, estudando efeitos tóxicos do boro, aplicou $0,56,112$ e 224 quilos de bórax por hectare, detectando, respectivamente, $29,135,162$, e $183 \mathrm{ppm}$ de $\mathrm{B}$ no meristema (na base de peso de material fresco).

Humbert e outros (12), estudando problemas de salinidade do solo e excesso de $B$ em relação à cana-de-açúcar com 14 meses, no Vale do Tambo, Peru, encontraram teores extremamente altos de $B$ no solo $(10,5 \mathrm{ppm})$ e na planta (folhas $3,4,5$ e $6,580 \mathrm{ppm})$; bainhas $(3,4,5$ e 6 , $160 \mathrm{ppm})$; e nas extremidades de crescimento $(180 \mathrm{ppm})$. Sintomas de toxicidade do $\mathrm{B}$ à cana podem ser esperados quando seu teor no solo estiver ao redor de $50 \mathrm{ppm}$. A distribuição de $B$ nâo é homogênea na folha da cana: os teores mais elevados $(890-1.850 \mathrm{ppm})$ ocorrem nos tecidos marginais mortos; os tecidos cloróticos apresentam $390-440 \mathrm{ppm}$, e os tecidos verdes, próximos à nervura central, 100-170 ppm. Estudando a aplicação controlada de boro à cana, em cinco solos, nas doses de 1,10 e $50 \mathrm{ppm}$, verificaram esses autores, para as doses mais elevadas, acentuada coloração avermelhada nas margens das folhas, além de fraca necrose. O excesso de B provoca sensível retardamento no crescimento da planta.

Entre nós, Haag (10) estudou, em soluções nutritivas, a nutrição mineral da cana var. CB 41-76. Na série sem boro obteve, em curto espaço de tempo, plantas com o crescimento paralisado, com morte do tecido apical. As folhas mais novas se apresentavam enroladas. Os colmos eram mais finos e internamente apresentavam estrias pardas, pouco abaixo do broto terminal. Sintomas foliares manifestaram-se nas folhas mais novas, com manchas, a princípio claras, pequenas, dispostas ao longo das nervuras.

Paralelamente ao estudo dos efeitos do boro na cana-de-açúcar em condições de campo (6), procedeu-se ao estudo desse elemento em condições controladas pelo emprego de vasos contendo solo pobre em boro. 


\section{2 - MATERIAL E MÉTOdos}

Como vasos, empregaram-se tambores metálicos, cortados ao meio, formando recipientes de 100 litros de capacidade, previamente limpos e pintados interna e externamente com tinta preta (Neutrol 45", tinta betuminosa fabricada por Otto Baumgart, São Paulo). Um orifício na parte lateral inferior do tambor permitia a drenagem do excesso de água de irrigação para um garrafão de cinco litros de capacidade, colocado em nivel inferior ao do fundo do vaso.

Como substrato, foi empregado solo da série Ibitiruna (15), com baixo teor em boro $(3)(0,10)$ ppm $B$ solúvel em água obtido pelo método de Dible, Truog e Berger (5). De local situado próximo ao ensaio de campo desta série (6), coletaram-se aproximadamente cinco toneladas de terra de uma camada superficial de $20 \mathrm{~cm}$. A terra foi seca ao ar, peneirada e homogeneizada como terra fina seca ao ar (TFSA), da qual foram colocados 80 litros em cada meio tambor, permanecendo os vasos a céu aberto. Pequena porção dessa terra constituiu o material para as determinações químicas usuais, cujos resultados foram os seguintes:

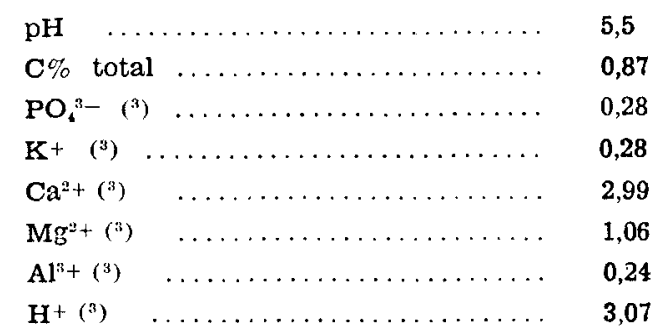

De um modo geral, trata-se de solo bem suprido de nutrientes, com exceção ao fósforo. O teste biológico do girassol (4) indícou ser esse solo ligeiramente deficiente ou não deficiente em boro (7).

Nesses vasos, em Janeiro de 1971 plantou-se cana da variedade CB 41-14 servindo de mudas pedaços de colmo com aproximadamente $2 \mathrm{~cm}$ e apresentando uma única gema. Inicialmente, foram colocadas quatro gemas em cada vaso e, após desbaste, ficaram apenas três. A germinação deu-se oito dias após.

Antes do plantio a terra de cada vaso recebeu adubação fosfatada uniforme, fornecida por fosfato monocálcico $\left(\mathrm{Ca}\left(\mathrm{H}_{2} \mathrm{PO}_{4}\right)_{2} \cdot \mathrm{H}_{2} \mathrm{O}\right)$, fosfato monoamônico $\left(\mathrm{NH}_{4} \mathrm{H}_{2} \mathrm{PO}_{4}\right)$ e fosfato monopotássico $\left(\mathrm{KH}_{2} \mathrm{PO}_{4}\right)$, mantendo-se no vaso $40 \mathrm{ppm}$ de $\mathrm{P}$ de cada fosfato, muito bem misturados à terra antes de colocá-la nos vasos.

Decorridos dois meses do plantio da cana, adicionou-se boro em niveis crescentes, na forma de ácido bórico $\left(\mathrm{H}_{3} \mathrm{BO}_{3}\right)$, e os tratamentos, com cinco repetições (cinco linhas de vasos, com $1,50 \mathrm{~m}$ de espaçamento) em delineamento de blocos casualizados foram os seguintes:
1. 0,0 ppm $B(O B)$
2. $0,5 \mathrm{ppm}$ B $(0,04 \mathrm{~g} /$ vaso $)$ (1B)
3. $1,0 \mathrm{ppm}$ B $(0,08 \mathrm{~g} /$ vaso $)$ (2B)
4. $2,0 \mathrm{ppm} \mathrm{B}(0,16 \mathrm{~g} /$ vaso $)$ (4B)
5. $3,0 \mathrm{ppm} \mathrm{B}(0,24 \mathrm{~g} /$ vaso $)(6 \mathrm{~B})$
6. $4,0 \mathrm{ppm} \mathrm{B}(0,32 \mathrm{~g} /$ vaso $)$ (8B)
7. $8,0 \mathrm{ppm} \mathrm{B}(0,64 \mathrm{~g} /$ vaso $)(16 \mathrm{~B})$

(3) Teor trocável em e.mg/100 g de solo. 
O tratamento $1 \mathrm{~B}(0,5 \mathrm{ppm} \mathrm{B})$ corresponde, no campo, à aplicação, aproximadamente, de $5 \mathrm{~kg} / \mathrm{ha}$ de bórax.

A seguir, completou-se a administração de nutrientes em várias aplicações. ficando, no final, as seguintes quantidades por vaso:

\begin{tabular}{|c|c|}
\hline Nitrogênio: & $9,6 \mathrm{~g} \mathrm{~N}(120 \mathrm{ppm})$, completado com $\mathrm{NH}_{4} \mathrm{NO}_{3}$ \\
\hline Fósforo: & $25.4 \mathrm{~g} \mathrm{P}_{2} \mathrm{O}_{5}(138 \mathrm{ppm} \mathrm{P})$, como $\mathrm{Ca}\left(\mathrm{H}_{2} \mathrm{PO}_{4}\right)_{2} \cdot \mathrm{H}_{2} \mathrm{O}$; \\
\hline Potássio: & $14,8 \mathrm{~g} \mathrm{~K}_{2} \mathrm{O}(154 \mathrm{ppm} \mathrm{K})$, completado com $\mathrm{K}_{2} \mathrm{SO}_{4}$; \\
\hline Cálcio: & $4,2 \mathrm{~g} \mathrm{CaO}(38 \mathrm{ppm} \mathrm{Ca})$, como $\mathrm{Ca}\left(\mathrm{H}_{2} \mathrm{PO}_{4}\right)_{2} \cdot \mathrm{H}_{2} \mathrm{O}$ \\
\hline Magnésio: & 2,0 g MgO (15 ppm Mg), como $\mathrm{MgSO}_{4} .7 \mathrm{H}_{2} \mathrm{O}$ \\
\hline Enxofre: & 5,1 g $S(63,7$ ppm $\mathrm{S})$, diversos; \\
\hline Cobre: & $0,28 \mathrm{mg} \mathrm{Cu}(0,004 \mathrm{ppm} \mathrm{Cu})$, como CuSO $.5 \mathrm{H}_{2} \mathrm{O}$; \\
\hline Molibdênio: & $0,51 \mathrm{mg}$ Mo $(0,006 \mathrm{ppm} \mathrm{Mo})$, como $\mathrm{H}_{2} \mathrm{MoO}_{4} \cdot \mathrm{H}_{2} \mathrm{O}$ \\
\hline Zinco: & $1,50 \mathrm{mg} \mathrm{Zn}(0,019 \mathrm{ppm} \mathrm{Zn})$, como $\mathrm{ZnSO}_{4} \cdot 7 \mathrm{H}_{2} \mathrm{O}$ \\
\hline Ferro: & $16,00 \mathrm{mg} F \mathrm{Fe}(0,200 \mathrm{ppm} \mathrm{Fe})$, como $\mathrm{FeSO}_{4} \cdot 7 \mathrm{H}_{2} \mathrm{O}$ \\
\hline Manganês: & $15,08 \mathrm{mg} \mathrm{Mn}(0,188 \mathrm{ppm} \mathrm{Mn})$, como $\mathrm{Mn} \mathrm{Cl}_{2}, 4 \mathrm{H}_{2} \mathrm{O}$; \\
\hline Cloro: & $19,45 \mathrm{mg} \mathrm{Cl}(0,243 \mathrm{ppm} \mathrm{Cl})$, como $\mathrm{MnCl}_{2}, 4 \mathrm{H}_{2} \mathrm{O}$. \\
\hline
\end{tabular}

Os micronutrientes foram fornecidos através da aplicaçăo de $30 \mathrm{ml}$ por vaso, da solução de Hoagland \& Arnon (11), à exceção de boro e ferro, que foram administrados separadamente.

Dois meses após a colheita da cana-planta fez-se a primeira aplicação dos mesmos nutrientes, dos mesmos compostos, utilizados na cana-planta, com exceção do boro, que não foi administrado, para o estudo do efeito residual desse elemento na cana-soca. Posteriormente foram feitas mais quatro aplicações, ficando, no final as seguintes quantidades por vaso:

\begin{tabular}{|c|c|}
\hline Nitrogênio: & $3.4 \mathrm{~g} \mathrm{~N}(42 \mathrm{ppm} \mathrm{N})$, como $\mathrm{NH}_{4} \mathrm{H}_{2} \mathrm{PO}_{4}$ e $\mathrm{NH}_{4} \mathrm{NO}_{3}$ \\
\hline Fósforo: & 16,4 g $\mathrm{P}_{2} \mathrm{O}_{5}(89 \mathrm{ppm} \mathrm{P})$, como $\mathrm{KH}_{2} \mathrm{PO}_{4}$ e $\mathrm{Ca}\left(\mathrm{H}_{2} \mathrm{PO}_{4}\right)_{2} \cdot \mathrm{H}_{2} \mathrm{O}$ \\
\hline Potássio: & $6,8 \mathrm{~g} \mathrm{~K}_{2} \mathrm{O}(71 \mathrm{ppm} \mathrm{K})$, como $\mathrm{KH}_{2} \mathrm{PO}_{4}$ e $\mathrm{K}_{2} \mathrm{SO}_{4}$ \\
\hline Cálcio: & $1,4 \mathrm{~g} \mathrm{CaO}(13 \mathrm{ppm} \mathrm{Ca})$, como $\mathrm{Ca}\left(\mathrm{H}_{2} \mathrm{PO}_{4}\right)_{2} \cdot \mathrm{H}_{2} \mathrm{O}$ \\
\hline Magnésio: & $2,0 \mathrm{~g} \mathrm{MgO}(15 \mathrm{ppm} \mathrm{Mg})$, como $\mathrm{Mg} \mathrm{SO} \cdot 7 \mathrm{H}_{2} \mathrm{O}$ \\
\hline Cobre: & $0,15 \mathrm{mg} \mathrm{Cu}(0,002 \mathrm{ppm} \mathrm{Cu})$, como $\mathrm{Cu} \mathrm{So}_{4} \cdot 5 \mathrm{H}_{2} \mathrm{O}$ \\
\hline Molibdênio: & $0,27 \mathrm{mg}$ Mo $(0,003 \mathrm{ppm} \mathrm{Mo})$, como $\mathrm{H}_{2} \mathrm{MoO}_{4} \cdot \mathrm{H}_{2} \mathrm{O}$ \\
\hline Zinco: & $0,80 \mathrm{mg} \mathrm{Zn}(0,010 \mathrm{ppm} \mathrm{Zn})$, como $\mathrm{Zn} \mathrm{SO} \cdot 7 \mathrm{H}_{2} \mathrm{O}$ \\
\hline Ferro: & $10,98 \mathrm{mg} \mathrm{Fe}(0,137 \mathrm{ppm} \mathrm{Fe})$, como $\mathrm{Fe} \mathrm{SO}_{4} .7 \mathrm{H}_{2} \mathrm{O}$ \\
\hline Manganês: & $8,04 \mathrm{mg} \mathrm{Mn}(0,100 \mathrm{ppm} \mathrm{Mn})$, como $\mathrm{Mn} \mathrm{Cl}_{2} .4 \mathrm{H}_{2} \mathrm{O}$ \\
\hline Cloro: & $10,37 \mathrm{mg} \mathrm{Cl}(0,130 \mathrm{ppm} \mathrm{Cl})$, como $\mathrm{Mn} \mathrm{Cl}_{2} .4 \mathrm{H}_{2} \mathrm{O}$ \\
\hline
\end{tabular}

As quantidades de macronutrientes administradas basearam-se em trabalho de Catani, citado por Malavolta e Haag (14), estimando-se em $10 \mathrm{~kg}$ a produção de cana de cada vaso. Para as regas, empregou-se água desmineralizada.

Aos cinco e aos oito meses de idade da cana-planta foram colhidas amostras de folhas para as determinaçōes de boro, tomando-se ao acaso três folhas inteiras (lâminas) de cada vaso, sem a nervura principal. Folhas das posições $+1,+3$ e -1 foram colhidas segundo critério de Kuijper, já descrito no trabalho anterior (6). Depois de secas a $65-70^{\circ} \mathrm{C}$, as folhas foram finamente moídas em moinho wiley de tamanho médio, de malha 20 e dotado de partes de aço inoxidável especial para trabalhos com micronutrientes. 
No material assim preparado e embalado em sacos plásticos analisou-se o boro pelo método colorimétrico da curcumina $(5,13)$.

As colheitas da cana foram realizadas aos doze meses de idade (cana-planta), e 12 meses mais tarde (cana-soca). Os colmos de cada vaso foram pesados e medidos em altura $(\mathrm{cm})$ e contou-se o número de entrenós de cada um.

Todas as canas produzidas em cada vaso serviram de amostra para determinaçōes do brix e polarização do caldo, através das quais calculou-se o açúcar-provável, da maneira descrita em trabalho anterior (6).

\section{3 - RESULTADOS E DISCUSSÃO}

Os dados médios de produção de cana-planta em vasos e um resumo dos resultados da análise de sua variância encontram-se no quadro 1.

Houve diferenças significativas dos tratamentos ao nível de $5 \%$ de probabilidade, pelo teste F: o tratamento $8 \mathrm{ppm}$ de B foi significativamente inferior aos demais, que não diferiram entre si.

$\mathrm{O}$ boro aplicado ao solo não proporcionou aumentos significativos na produção de cana em vasos, e induziu efeito depressivo, na dose mais elevada ( $8 \mathrm{ppm})$, comparada com o tratamento adubação básica completa menos boro (O ppm B) (figuras 1 e 2 ).

O quadro 2 apresenta dados ilustrativos, obtidos no presente ensaio. Verifica-se, por eles, que não houve variação quando se modificou a dose

QUADRO 1. - Produçōes de cana-planta da variedade CB 41-14, obtidas em ensaio de aplicação de doses crescentes de $B$ a solo da série Ibitiruna, do município de Piracicaba, SP, em vasos

\begin{tabular}{c|c|c}
\hline TRATAMENTO & PRODUÇO MEDIA & d.m.s. (DUNCAN, 5\%) \\
\hline ppm B & kg/vaso & $\mathbf{k g} / \mathbf{v a s o}$ \\
0,0 & 6,664 & $\mathrm{D} 7=0,870$ \\
0,5 & 6,684 & $\mathrm{D} 6=0,862$ \\
1,0 & 6,394 & $\mathrm{D} 5=0,846$ \\
2,0 & 6,632 & $\mathrm{D} 4=0,828$ \\
3,0 & 6,748 & $\mathrm{D} 3=0,807$ \\
4,0 & 6,466 & $\mathrm{D} 2=0,767$ \\
8,0 & 5,340 & \\
\hline F & $3,50 *$ & \\
s $(\hat{\mathrm{m}})$ & 0,263 & \\
C. V. \% & 9,2 & \\
\hline
\end{tabular}

* significativo a $5 \%$ 
Vol. 35, N. ${ }^{2} 23$

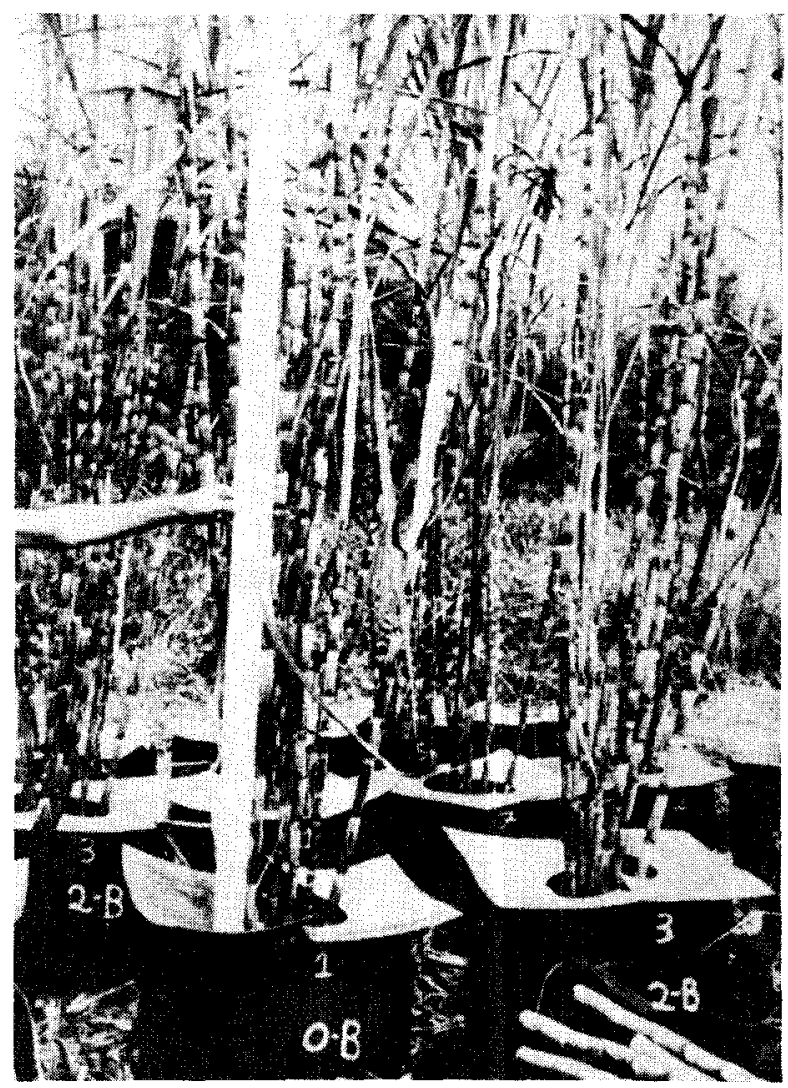

Figura 1. - Ensaio com cana-de-açúcar var. CB 41/14, em vasos com solo da série Ibitiruna que recebeu doses crescentes de boro.

de boro, à exceção da dose máxima estudada $(8 \mathrm{ppm})$ que, em todos os casos, parece ter sido prejudicial à cana.

As produções médias de cana-soca em vasos e um resumo da respectiva análise de variância encontram-se no quadro 3 .

$O$ teste $F$ mostrou que não houve diferenças significativas entre os tratamentos estudados.

$\mathrm{O}$ boro aplicado à cana-planta não proporcionou aumentos significativos na produção de cana-soca e não induziu efeito depressivo pelas doses mais elevadas. Duas produções sucessivas de cana (planta e soca) não foram afetadas pela não administração desse elemento. 


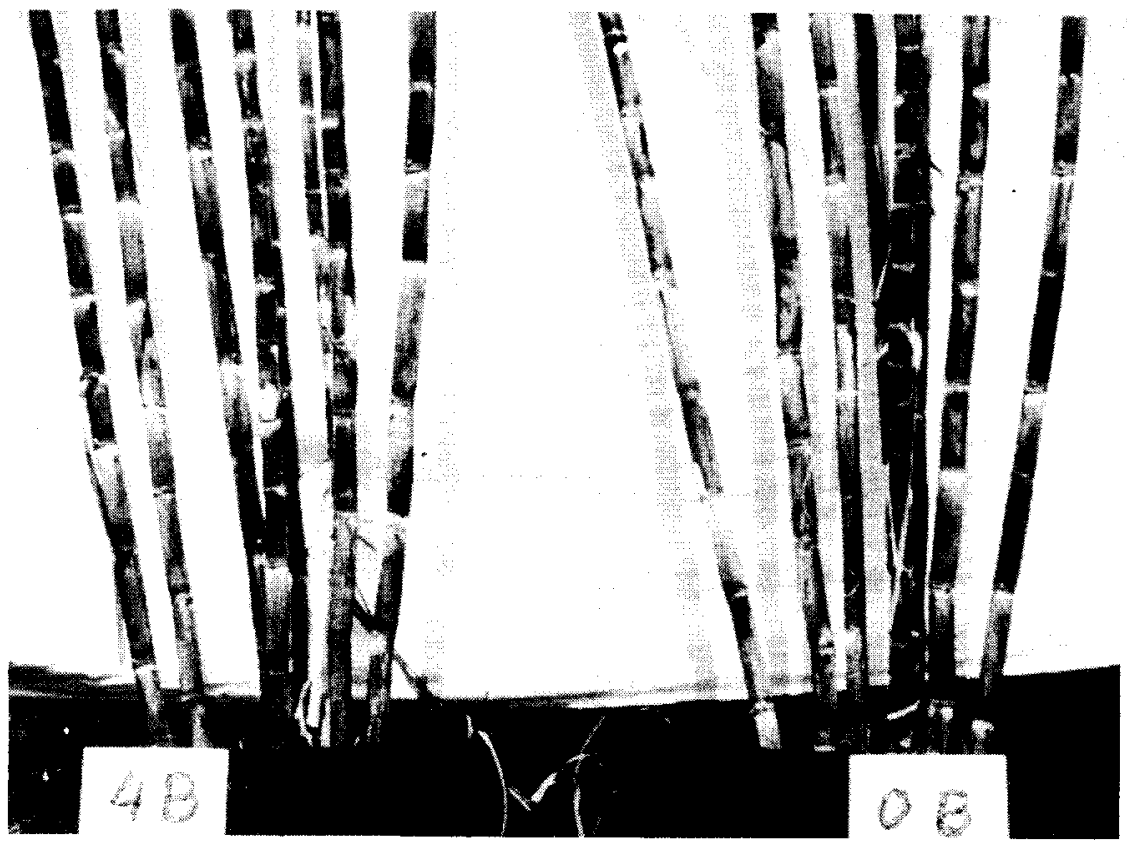

Figura 2. - Tratamentos 4-B e 0-B, do ensaio com cana-de-açúcar var. CB 41/14, em vasos com solo da série Ibitiruna que recebeu doses crescentes de boto.

QUADRO 2. - Dados de ensaio em vasos, com cana variedade CB 41-14 em solo da série Ibitiruna que recebeu doses crescentes de boro

\begin{tabular}{c|c|c|c|c}
\hline \multirow{2}{*}{ TRATAMENTO } & \multicolumn{4}{|c}{ COLMO } \\
\cline { 2 - 5 } & $\begin{array}{c}\text { N.v médio } \\
\text { por vaso }\end{array}$ & Peso médio & $\begin{array}{c}\text { Comprimento } \\
\text { médio }\end{array}$ & $\begin{array}{c}\text { Comprimento } \\
\text { médio dos } \\
\text { entrenós }\end{array}$ \\
\hline ppm B & & $\mathrm{g}$ & $\mathrm{m}$ & $\mathrm{cm}$ \\
0,0 & 8,4 & 793 & 1,59 & 8,1 \\
0,5 & 8,0 & 798 & 1,63 & 8,0 \\
1,0 & 8,2 & 780 & 1,55 & 8,1 \\
2,0 & 8,8 & 754 & 1,60 & 7,9 \\
3,0 & 8,0 & 843 & 1,61 & 7,9 \\
4,0 & 8,0 & 808 & 1,66 & 7,9 \\
8,0 & 7,4 & 722 & 1,52 & 7,6 \\
\hline
\end{tabular}


QUADRO 3. - Produções médias de cana-soca da variedade CB 41-14, obtidas em ensaio de aplicação de doses crescentes de boro à cana-planta cultivada em vasos com solo da série Ibitiruna

\begin{tabular}{c|c}
\hline TRATAMENTO & PRODUÇA MEDIA \\
\hline ppm B & $\mathbf{k g} / \mathbf{v a s o}$ \\
0,0 & 3,750 \\
0,5 & 3,316 \\
1,0 & 3,268 \\
2,0 & 3,530 \\
3,0 & 3,106 \\
4,0 & 3,272 \\
8,0 & 3,286 \\
F & 0,96 n.s. \\
s ( $\hat{m})$ & 0,219 \\
C. V. $\%$ & 14,6 \\
\hline
\end{tabular}

n.s. não significativo

O teste do girassol (7) mostrou ser essa a série menos suprida em boro.

Ao que tudo indica, a cana-de-açúcar é pouco exigente no micronutriente em estudo, sendo-lhe suficientes para desenvolvimento e produção normais, as quantidades mínimas existentes no solo e na própria muda $\left(^{4}\right)$.

O quadro 4 apresenta os dados médios de produção de açúcar-provável na cana-planta, calculados a partir dos índices Brix e de polarização do caldo, um resumo dos resultados da análise de variância de tais dados, e as diferenças mínimas significativas (Duncan, 5\%) para comparação das médias de produção de açúcar-provável.

Verifica-se pelo teste $F$, que houve diferenças significativas entre os tratamentos, ao nível de $5 \%$ de probabilidade.

O tratamento $8 \mathrm{ppm}$ de $\mathrm{B}$ foi estatisticamente inferior ao tratamento O ppm e ao $2 \mathrm{ppm}$ (média mais elevada), e o tratamento $4 \mathrm{ppm}$ foi inferior a este último. Os demais não diferiram entre si.

Houve decréscimo no açúcar-provável do caldo, pela aplicação da dose mais elevada de boro $(8 \mathrm{ppm})$, em relação à testemunha $(\mathrm{O} \mathrm{ppm})$. Entretanto, não houve aumentos do açúcar-provável pela administração do boro ao solo, relativamente à adubação básica completa menos boro (O ppm B). Esses resultados concordam com os de produção de cana.

(9) Evans (8), analisando a cana, encontrou $1,5 \mathrm{ppm}$ de $B$ nos nós e um pouco mais nas regióes do colmo a eles adjacentes. 
QUADRO 4. - Dados médios de açúcar-provável para cana-planta var. CB 41/14, obtidos em ensaio em vasos para verificação de efeitos da aplicação de boro a solo da série Ibitiruna, do município de Piracicaba, SP

\begin{tabular}{c|c|c}
\hline TRATAMENTO & AÇÚCAR-PROVAVEL & d.m.S. (DUNCAN, 5\%) \\
\hline ppm B & kg/t cana & \\
0,0 & 114,0 & $\mathrm{D} 7=8,3$ \\
0,5 & 110,5 & $\mathrm{D} 6=8,2$ \\
1,0 & 111,1 & $\mathrm{D} 5=8,1$ \\
2,0 & 115,5 & $\mathrm{D} 4=7,9$ \\
3,0 & 110,6 & $\mathrm{D} \mathrm{3}=7,7$ \\
4,0 & 106,1 & $\mathrm{D} 2=7,3$ \\
8,0 & 103,6 & \\
\hline F & $2,80 *$ & \\
s $(\hat{\mathrm{m}})$ & 2,5 & \\
C. V. $\%$ & 5,1 & \\
\hline
\end{tabular}

* significativo a $5 \%$

No quadro 5 constam os dados médios calculados de açúcar-provável, obtidos da cana-soca e um resumo da análise de variância desses dados.

Pelo teste $F$ não houve diferenças significativas entre os tratamentos.

Não houve, portanto, aumentos do açúcar-provável pela administração de boro ao solo, na cana-planta, o que está de acordo com os resultados da cana-planta. Não houve, por outro lado, decréscimo pela administração da dose mais elevada de boro ( $8 \mathrm{ppm})$, como aconteceu na cana-planta.

Os teores médios de boro determinados nas folhas $+3,+1 \mathrm{e}-1$, aos cinco meses de idade (1. a amostragem), encontram-se no quadro 6 , e os dados da análise de variância correspondente, no quadro 7 .

$O$ teste $F$ revelou diferenças significativas entre os tratamentos, entre as posições das folhas, e interação tratamentos $\mathrm{x}$ folhas, ao nível de $1 \%$ de probabilidade.

Os valores das diferenças mínimas significativas (Duncan, 5\%) para comparação das médias dos tratamentos e das posições das folhas estudadas constam do quadro 6 .

Independente das três posiçôes estudadas, os tratamentos $8 \mathrm{ppm}$ e $4 \mathrm{ppm}$ diferiram entre si e foram superiores aos demais. O tratamento $3 \mathrm{pmm}$, não diferindo do $2 \mathrm{ppm}$, foi superior aos tratamentos em que a dose de boro foi mais baixa. O tratamento $2 \mathrm{ppm}$, não diferindo de $0,5 \mathrm{ppm}$ c 1 ppm, foi superior à testemunha sem boro $(\mathrm{O}$ ppm B), que não diferiu 
QUADRO 5. - Dados médios de açúcar-provável para cana-soca variedade CB 41-14, obtidos em ensaio em vasos com solo da série Ibitiruna, para verificação do efeito residual do boro aplicado na cana-planta

\begin{tabular}{c|c}
\hline TRATAMENTO & AÇÚCAR-PROVAVEL \\
\hline ppm B & $\mathbf{k g} / \mathbf{t}$ cana \\
0,0 & 96,3 \\
0,5 & 94,6 \\
1,0 & 95,4 \\
2,0 & 97,9 \\
3,0 & 93,0 \\
4,0 & 92,8 \\
8,0 & 96,4 \\
F & 0,49 n.s. \\
s ( $\hat{\mathbf{m}})$ & 2,7 \\
C. v. $\%$ & 6,3 \\
\hline
\end{tabular}

n.s. não significativo

QUADRO 6. - Teores médios de boro (ppm), em folhas de diferentes posiçōes em cana-planta var. CB 41-14 com cinco meses de idade (1. a amostragem), vegetando em vasos para verificação de efeitos da aplicação de boro em solo da série Ibitiruna do município de Piracicaba, SP

\begin{tabular}{|c|c|c|c|c|c|}
\hline \multirow{2}{*}{ TRATAMENTO } & \multicolumn{4}{|c|}{ FOLHA } & \multirow{2}{*}{ d.m.s. (*) } \\
\hline & +3 & +1 & -1 & Média & \\
\hline \multicolumn{6}{|l|}{ ppm $B$} \\
\hline 0,0 & 84,7 & 74,8 & 92,9 & 84,1 & $\mathrm{D} 7=14,0$ \\
\hline 0,5 & 96,8 & 85,4 & 96,0 & 92,7 & D $6=13,8$ \\
\hline 1,0 & 100,3 & 84,6 & 90,5 & 91,8 & $\mathbf{D} 5=13,6$ \\
\hline 2,0 & 104,6 & 98,1 & 104,9 & 102,5 & $\mathrm{D} 4=13,3$ \\
\hline 3,0 & 122,7 & 101,2 & 111,6 & 111,8 & $\mathbf{D} 3=12,9$ \\
\hline 4,0 & 150,8 & 144,8 & 115,0 & 138,2 & $\mathrm{D} 2=12,2$ \\
\hline 8,0 & 264,0 & 289,0 & 195,0 & 249,3 & \\
\hline Médias & 132,0 & 126,0 & 115,1 & 124,4 & \\
\hline $\mathbf{s}(\hat{\mathbf{m}})$ & \multicolumn{4}{|c|}{2,8} & \\
\hline d.m.s. (*) & \multicolumn{2}{|c|}{$\mathrm{D} 3=8,3$} & \multicolumn{2}{|c|}{$\mathrm{D} 2=7,9$} & \\
\hline
\end{tabular}

(*) Duncan, 5\% 
QUADRO 7. - Análise da variância dos dados do quadro 6

\begin{tabular}{l|r|r|c}
\hline \multicolumn{1}{c|}{$\begin{array}{l}\text { FONTE DE } \\
\text { VARIAÇO }\end{array}$} & G.L. & Q.M. & F \\
\hline Tratamentos (T) & 6 & $50.304,46$ & $177,99 * *$ \\
Folhas (F) & 2 & $2.555,24$ & $9,04 * *$ \\
Int. T x F & 12 & $2.149,88$ & $7,61 * *$ \\
Blocos & 4 & 408,70 & 1,45 n.s. \\
Resíduo & 80 & 282,62 & \\
\hline
\end{tabular}

C.V. $\%$

13,5

** significativo a $1 \%$

n.s. nāo significativo

de 0,5 e $1 \mathrm{ppm}$. As doses de 0,5 e $1 \mathrm{ppm} \mathrm{B}$ não provocaram aumentos nos teores de boro nas folhas, aumentando, contudo, na dose $2 \mathrm{ppm}$, em relação a $0 \mathrm{ppm}$. De 2 para $3 \mathrm{ppm}$ não houve aumento, o que ocorreu nas doses de 4 e 8 ppm.

$\mathrm{Na}$ comparação das médias das três posições de folhas, o teor de boro na folha de posição $-1(115,1 \mathrm{ppm})$ foi estatisticamente inferior aos teores das folhas $+3(132 \mathrm{ppm}) \mathrm{e}+1(126 \mathrm{ppm})$, que não diferiram entre si, ao nível de $5 \%$ de probabilidade.

As três posições de folhas utilizadas mostraram-se eficientes em relação às doses de boro aplicadas ao solo; todavia, as de posições $+3 \mathrm{e}$ +1 revelaram valores estatisticamente superiores aos de -1 .

Não foram observados sintomas de deficiência de boro na cana que vegetou nos vasos no tratamento $O$ ppm $B$ (2), nem sintomas de toxicidade nas doses mais elevadas desse micronutriente. Humbert e outros (12) relatam que sintomas de toxicidade de boro à cana só podem ser esperados quando seu teor no solo estiver ao redor de $50 \mathrm{ppm}$.

Pesquisas realizadas com cana vegetando em soluções nutritivas revelaram boas produções de colmos quando a taxa de boro nas folhas alcançou $1 \mathrm{ppm} \mathrm{(8).} \mathrm{Os} \mathrm{valores} \mathrm{encontrados} \mathrm{no} \mathrm{presente} \mathrm{trabalho} \mathrm{estão} \mathrm{muito}$ acima desse valor, mesmo nos tratamentos não adubados.

Observa-se pelo quadro 6 que os teores de B encontrados nas folhas da cana, nos vasos que receberam as maiores doses do elemento, foram bastante altos, mas estão de acordo com os dados de Humbert e outros (12), 
que encontraram $580 \mathrm{ppm}$ de $\mathbf{B}$ nas folhas $3,4,5$ e 6 , num solo (sem adição de B) que continha $10,5 \mathrm{ppm}$, no Peru. Esses autores obtiveram até $1.850 \mathrm{ppm} \mathrm{B}$ nas folhas da cana-de-açúcar. Também Bowen (2) obteve teores altos de $B$.

Não obstante ser de $0,10 \mathrm{ppm}$ o teor natural de boro no solo da série Ibitiruna utilizado no ensaio em vasos, teor esse considerado baixo, foram muito altos os teores de boro encontrados nas folhas, não se podendo esperar, portanto, respostas da aplicação de boro ao solo na produção de cana.

No quadro 8 encontram-se os teores médios de boro determinados nas folhas $+3,+1$ e -1 , aos oito meses de idade da cana do ensaio em vasos (2. ${ }^{\mathrm{a}}$ amostragem) e, no quadro 9 , os resultados da análise de variância correspondente.

$O$ teste $F$ não revelou diferenças significativas entre tratamentos, posições das folhas e interação tratamentos $\mathrm{x}$ folhas.

Os teores de $\mathrm{B}$ encontrados nessa segunda amostragem (média geral de $61,4 \mathrm{ppm}$ ) foram bem menores que os da primeira (média geral 124,4 ppm) e não seletivos em relação às doses de boro aplicada.

QUADRO 8. - Teores médios de boro (ppm) em folhas de diferentes posiçōes, em cana-de-açúcar var. CB 41-14 com oito meses de idade (2. amostragem), vegetando em vasos com solo da série Ibitiruna, para verificaçāo de efeitos da aplicação de boro em solos do município de Piracicaba, SP

\begin{tabular}{c|c|c|c|c}
\hline \multirow{2}{*}{ TRATAMENTO } & \multicolumn{4}{|c}{ FOLHA } \\
\cline { 2 - 5 } & +3 & +1 & -1 & Média \\
\hline \multirow{2}{*}{ ppm B } & & & & \\
0,0 & 48,3 & 55,1 & 59,8 & 54,4 \\
0,5 & 57,8 & 50,4 & 56,0 & 54,7 \\
1,0 & 63,4 & 56,9 & 52,2 & 57,5 \\
2,0 & 61,6 & 57,2 & 48,7 & 55,8 \\
3,0 & 62,6 & 71,7 & 65,7 & 66,7 \\
4,0 & 68,9 & 60,7 & 68,3 & 66,0 \\
8,0 & 86,9 & 77,4 & 59,9 & 74,7 \\
\hline Média & 64,2 & 61,3 & 58,7 & 61,4 \\
\hline
\end{tabular}


QUADRO 9. - Análise da variância dos dados do quadro 8

\begin{tabular}{l|c|c|c}
\hline $\begin{array}{r}\text { FONTE DE } \\
\text { VARIACAO }\end{array}$ & G.L. & Q.M. & F \\
\hline Tratamentos (T) & 6 & 915,03 & 2,14 n.s. \\
Folhas (F) & 2 & 270,31 & 0,63 n.s. \\
Int. T x F & 12 & 248,98 & 0,58 n.s. \\
Blocos & 4 & 416,50 & 0,98 n.s. \\
Residuo & 80 & 428,17 & \\
\hline C.V. \% & & & 33,7 \\
\hline
\end{tabular}

n.s. não significativo

\section{4 - CONCLUSÕES}

a) O boro aplicado a solo da série Ibitiruna, em vasos e apenas na cana-planta variedade CB 41-14, não proporcionou aumentos nas produções de cana e de açúcar-provável, tanto em cana-planta como em cana-soca. Induziu, entretanto, pela dose mais elevada (8 ppm B) efeitos dẹpressivos na cana-planta, em ambas as características mencionadas.

b) Os teores foliares de boro cresceram com as doses adicionadas ao solo, alcançando, aos cinco meses de idade da cana-planta, níveis muito elevados no tratamento de $8 \mathrm{ppm} \mathrm{B},(289 \mathrm{ppm})$. Esta foi a melhor época de amostragem de folhas (média geral de $124,4 \mathrm{ppm} \mathrm{B}$ ), sendo que a de oito meses mostrou valores bem mais baixos (média geral de 61,4 ppm B) e não seletivos em relação à dose de boro aplicada.

c) Com referência à posição da folha na planta, as três utilizadas mostraram-se eficientes em relação às doses de boro aplicadas. As folhas $+3 \mathrm{e}+1$ deram valores mais altos do que as folhas -1 .

EFFECTS OF BORON ON SUGARCANE CULTIVATED IN POTS WITH SOIL

\section{SUMMARY}

The effects of boron on the behaviour of sugarcane (plant-crop and first ratton crop) var. CB 41-14 were studied in a trial pot with increasing doses of this micronutrient to Ibitiruna soil from Piracicaba, SP, considered poor in boron content.

The boron applied did not increase cane growth nor sugar content, but $8 \mathrm{ppm} B$ reduced both cane and sugar production. Chemical analysis with leaves 5 months old revealed increasing boron contents according to the amounts given 
to the soil. With leaves 8 months old, the B levels were lower and non selective with respect to the boron amounts applied. The best time to sample leaves for $B$ analysis seems to be 5 months, in the conditions of the study.

\section{LITERATURA CITADA}

1. AYRES, A. S. Calcium silicate slag as a growth stimulant for sugar cane on low silicon soils. Soil Sci. $101(3): 216-227,1966$.

2. BOWEN, J. E. Some physiological effects of variable boron and zinc levels on sugar cane. Sugar News 45 (4):205-209, 1969.

3. BRASIL SOBRINHO, M. O. C. Levantamento do teor de boro em alguns solos do Estado de São Paulo. Piracicaba, ESALQ, USṔ, 1965.135 p. (Tese de livre-docência, mimeog.)

4. COLWELL, W. E. A biological method for determining the relative boron contents of soils. Soil Sci. 56:71-94, 1943.

5. DIBLE, W. T.; TRUOG, E. \& BERGER, K. C. Boron determination in soils and plants. Simplified curcumin procedure. Anal. Chemistry 26:418-421, 1954.

6. ESPIRONELO, A.; BRASIL SOBRINHO, M. O. C. \& IGUE, T. Efeitos do boro em cana-de-açúcar cultivada em alguns solos do município de Piracicaba. I. Cana-planta. Bragantia 35:191-211, 1976.

7. —__ _ _ _ Avaliação do boro assimilável e provas de respostas, pelo método biológico do girassol, à administração desse elemento a alguns solos cultivados com cana-de-açúcar. Bragantia 35: 221-236, 1976.

8. EVANS, H. Elements other than nitrogen, potassium, and phosphorus in the mineral nutrition of sugar cane. Proc. 10th Congr. ISSCT. Hawaii, 1959. p.473-508.

9. GALLO, J. R.; ALVAREZ, R. \& ABRAMIDES, E. Amostragem em cana-de-açúcar, para fins de análise foliar. Bragantia 21:899-921, 1962.

10. HAAG, H. P. Estudos de nutrição mineral na cana-de-açúcar (Saccharum officinarum, L.) variedade CB 41-76 cultivada em solução nutritiva. Piracicaba, ESALQ, $1965.141 \mathrm{p}$. (Tese de livre-docência, mimeog.).

11. HOAGLAND, D. R. \& ARNON, D. I. The water-culture method for growing plants without soil. Berkeley, Univ. California agric. Exp. Sta., 1938. 39p. (Circular 347)

12. HUMBERT, R. P.; BAHME, R. B.; FERNANDES, E. D. \& THOMPSON, L. I. Overcoming boron and salinity problems in growing sugar cane in the Tambo Valley of Peru. Sugar Y Azucar 65:32-34, 1970.

13. LOTT, W. L.; McClUNG, A. C.; VITTA, R. de \& GALlO, J. R. Levantamento de cafezais pela análise foliar, em São Paulo e Paraná. São Paulo, IBEC Res. Institute, 1961, 69p. (Boletim 26)

14. MALAVOLTA, E. \& HAAG, H. P. Nutriçāo e adubação. In: Inst. bras. de Potassa, Ed. Cultur ae adubação da cana-de-açúcar. São Paulo. Inst. bras. Potassa. p. 237-278, 1964.

15. RANZANI, G.; FREIRE, O. \& KINJO, T. Carta de solos do município de Piracicaba. Piracicaba, ESALQ, Centro de Estudos de Solos, 1966. 64p.

16. SCHUSTER, C. E. \& STEPHENSON, R. E. Sunflower as an indicator plant of $B$ deficiency in soils. J. Amer. Soc. Agron. $32: 607-621,1940$.

17. TANADA, T. \& DEAN, L. A. Boron in some Hawaiian soils and crops. Hawaiian Planters'Record 46:65-74, 1942.

18. VAN DILLEWIJN, C. Botany of sugar cane. Massachussets, Walthan, Chr. Bot., 1952. 371p. 